\title{
Is There an Increased Arterial Stiffness in Patients with Primary Sjögren's Syndrome?
}

\author{
Meltem Sezis Demirci ${ }^{1}$, Gonca Karabulut ${ }^{2}$, Ozkan Gungor ${ }^{3}$, Aygul Celtik ${ }^{1}$, \\ Ercan $\mathrm{Ok}^{1}$ and Yasemin Kabasakal ${ }^{2}$
}

\begin{abstract}
Objective Primary Sjögren's syndrome (pSS) is a common chronic autoimmune disease that primarily affects the salivary and lacrimal glands. Arterial stiffness is one of the earliest detectable manifestations of adverse structural and functional changes within the vessel wall. The aim of this study was to evaluate the relationship between arterial stiffness and pSS.

Methods In this study, 75 female patients with pSS who fulfilled the American European Consensus Criteria for Sjögren's syndrome, were included. A total of 68 age-, sex- and body mass index-matched subjects were recruited as the control population. Arterial stiffness was assessed by measurement of the carotidfemoral pulse wave velocity (PWV).

Results The mean age of the patients was 54.0 \pm 9.3 years and the median duration of the disease was 10 years. Compared with the control subjects, patients with pSS had a higher mean PWV $(8.2 \pm 1.5 \mathrm{~m} / \mathrm{s}$ vs. $7.5 \pm$ $1.4 \mathrm{~m} / \mathrm{s} ; \mathrm{p}=0.01$ ). Correlation analysis showed that the PWV was positively correlated with age, body mass index, serum cholesterol, low-density lipoprotein (LDL) and C-reactive protein levels, blood pressure, mean arterial pressure (MAP), pulse pressure and left ventricular mass index. A multiple linear regression analysis revealed that arterial stiffness was associated with age, MAP and LDL levels in pSS patients.

Conclusion Although patients with pSS appear to have increased arterial stiffness, risk factors associated with arterial stiffness in these patients are similar to the general population. However, we cannot exclude the possibility that a higher PWV in pSS patients is caused, not by pSS itself, but by the use of steroids, hypertension and dyslipidemia.
\end{abstract}

Key words: primary Sjögren's syndrome, arterial stiffness, cardiovascular disease

(Intern Med 55: 455-459, 2016)

(DOI: 10.2169/internalmedicine.55.3472)

\section{Introduction}

In patients with arterial stiffness, the forward pulse wave travels faster, and the arterial waves reflected from the periphery reach the heart early during systole, which leads to higher systolic but lower diastolic blood pressure, an augmentation of the cardiac workload and a decrease of the coronary perfusion pressure. The aortic pulse wave velocity (PWV) reflects central arterial stiffness. The PWV is a predictor of cardiovascular outcome in patients with hypertension, diabetes, end-stage renal disease and in elderly hospi- talized subjects (1-4). PWV is an easier, more applicable, noninvasive and reproducible marker of subclinical atherosclerosis in rheumatology. An increased PWV has been detected in patients with rheumatologic diseases such as rheumatoid arthritis (RA) $(5,6)$, ankylosing spondylitis (7), scleroderma and polymyositis (8).

Primary Sjögren's syndrome (pSS) is a slowly progressive, inflammatory, systemic autoimmune disease affecting primarily the exocrine glands. The disease is characterized histologically by lymphocytic infiltration of the affected glands and clinically by the imaging of ocular and oral dryness (Sicca syndrome). According to the American European

${ }^{1}$ Department of Nephrology, Ege University Faculty of Medicine, Turkey, ${ }^{2}$ Department of Rheumatology, Ege University Faculty of Medicine, Turkey and ${ }^{3}$ Department of Nephrology, Kahramanmaras State Hospital, Turkey

Received for publication June 13, 2014; Accepted for publication March 15, 2015

Correspondence to Dr. Meltem Sezis Demirci, meltem.sezis@ege.edu.tr 
Consensus Criteria, the estimated prevalence rate of pSS is 0.1 to $0.6 \%$, with a preponderance of female over male patients at a ratio of 9:1 (9).

Primary Sjögren's syndrome shares clinical and serologic features with rheumatoid arthritis and systemic lupus erythematosus (SLE), two diseases characterized by acceleration of atherosclerosis. Although cardiac involvement in patients with Sjögren's syndrome is rarer than in patients with other rheumatic diseases such as RA and SLE, asymptomatic pericarditis and left ventricular diastolic dysfunction are common clinic entities (10). The association between pSS and atherosclerosis is unclear. Recently, Gerli et al. reported that flow-mediated dilatation rates of patients with pSS were similar to that of a control group, while nitrate-mediated vasodilation rates were lower in patients with pSS (11). Another study showed that an increased carotid artery intimamedia thickness (a sign of subclinical atherosclerosis) is associated with the presence of anti- Sjögren's-syndromerelated antigen A (anti-SSA) and leukopenia in patients with Sjögren's syndrome (12). To our knowledge, there are no studies investigating the effects of pSS on arterial stiffness. The aim of this study was to evaluate the relationship between arterial stiffness and pSS.

\section{Materials and Methods}

This study examined 75 female patients who fulfilled the US-European Consensus Criteria for pSS. This study was approved by the local Ethics Committee and an informed consent form was obtained from all subjects. The main objective of this study was to compare the arterial stiffness of pSS patients without a history of pre-existing cardiovascular disease to that of control subjects. Patients with persistent arrhythmia, advanced congestive heart failure (New York Heart Association III to IV stages), a recent history of myocardial infarction or stroke within six months, unstable angina pectoris, arrhythmia, renal dysfunction, diabetes mellitus or known glucose metabolism disturbances, current use of metformin or acarbose, or who were suspected of having another inflammatory disease, were excluded. The proportion of patients who were on glucocorticosteroid treatment was $13.7 \%$ and $9.6 \%$ of the patients were on immunosuppressive treatment. A total of 68 control subjects were recruited from among the age-, sex- and body mass index (BMI)-matched population. Demographic data were recorded from patient charts.

\section{Blood chemistry}

Blood samples under fasting conditions were obtained using uniform techniques before starting arterial stiffness measurements. Erythrocyte sedimentation rate and serum levels of urea, creatinine, sodium, potassium, hemoglobin, albumin, high sensitive C-reactive protein (CRP), cholesterol, triglycerides, high-density lipoprotein (HDL) and lowdensity lipoprotein (LDL) were measured. Blood chemistry parameters were assayed by standardized and automated techniques in the hospital's analytical laboratory, which adheres to several external quality control programs. In addition to these tests, several other rheumatologic tests were performed, including the measurement of antinuclear antibody (ANA) by using Hep-2 cells, Anti-Ro and anti-La antibodies by solid-phase enzyme-linked immunosorbent assay (ELISA) and rheumatoid factor (RF) measured by nephelometry.

\section{Echocardiographic measurements}

Echocardiographical measurements were performed according to the American Society of Echocardiography recommendations. The following assessments were recorded: left atrium diameter, left ventricular end-diastolic and endsystolic diameters, right ventricular end-diastolic diameter, and thickness of the posterior wall and the interventricular septum. The left ventricular mass (LVM) was calculated using the Devereux formula. The left ventricular mass index was calculated by dividing LVM by the body surface area. Intra-operator variability was less than five percent.

\section{Arterial stiffness measurements}

Arterial stiffness was assessed by carotid-femoral PWV with the SphygmoCor device (Atcor Medical, Sydney, Australia). Assessments were done by sequential recordings of the arterial pressure wave at the carotid and femoral arteries, and by measurement of the distance from the carotid sampling site to the suprasternal notch and from the suprasternal notch to the femoral sampling site. With a simultaneous electrocardiography (ECG) recording of the R-wave as reference, the integral software calculated the pulse wave transit time. Measurements were performed in fasting status after five to 10 minutes of resting. If used, antihypertensive medications were stopped at least 24 hours prior to evaluation. Arterial stiffness was measured by the same operator and intra-operator variability was less than five percent.

\section{Statistical analysis}

All parameters were expressed as the mean \pm SD. A p value of less than 0.05 was considered statistically significant. Comparisons between two groups were assessed by an independent t-test analysis. The Pearson's rank correlation was used to assess correlation of arterial stiffness with other variables. A multivariate stepwise linear regression analysis was used to define independent predictors of arterial stiffness in pSS patients. The software SPSS v.18 (SPSS, Chicago, USA) was used for all analyses.

\section{Results}

Across the whole group of patients, the mean age was $54.0 \pm 9.3$ (range 27-78) years, the median duration of the disease was 10 years with a range of one to 23 years, $15.4 \%$ of the patients used antihypertensive medication and $14.9 \%$ of the patients were smokers. Extra-glandular involvement was found in $41.7 \%$ of the patients. Mean systolic blood 
Table 1. Demographical Characteristic of Patients.

\begin{tabular}{lc}
\hline Variables & Mean \pm SD \\
\hline Age (years) & $54.0 \pm 9.3$ \\
Duration of disease(year) (median) & 10 \\
Extraglandular involvement(\%) & 41.7 \\
Antihypertensive drug use (\%) & 15.4 \\
Statin use (\%) & 5.5 \\
Smoking (\%) & 14.9 \\
BMI (kg/m $\left.{ }^{2}\right)$ & $28.9 \pm 4.8$ \\
SBP(mmHg) & $122 \pm 16$ \\
DBP(mmHg) & $76 \pm 9$ \\
LVMI (g/m ${ }^{2}$ ) (n=47) & $75 \pm 29$ \\
Mean cf-PWV (m/s) & $8.2 \pm 1.5$ \\
ANA positivity (\%) & 77,8 \\
-granular & 91,2 \\
-nucleolar & 3,5 \\
-homogen & 3,5 \\
-cytoplasmic & 1,8 \\
Anti-Ro/-La positivity (\%) & 69,3 \\
RF positivity (\%) & 47,1 \\
\hline SBP: systolic blood pressure, DBP: diastolic blood \\
pressure, LVMI: left ventricular mass \\
antinuclear antibodies, RF: rheumatoid factor \\
\end{tabular}

Table 3. Associations between Carotid Femoral PWV and Demographic and Laboratory Parameters in Patients with PSS.

\begin{tabular}{|c|c|c|}
\hline Parameters & $\mathbf{r}$ & p \\
\hline Age & 0.571 & 0.000 \\
\hline BMI & 0.380 & 0.001 \\
\hline Total cholesterol & 0.357 & 0.006 \\
\hline LDL cholesterol & 0.355 & 0.006 \\
\hline SBP & 0.503 & 0.000 \\
\hline DBP & 0.458 & 0.000 \\
\hline MAP & 0.494 & 0.000 \\
\hline PP & 0.346 & 0.010 \\
\hline LVMI & 0.300 & 0.040 \\
\hline $\log C R P$ & 0.315 & 0.017 \\
\hline $\mathrm{WBC}\left(\mathrm{mm} /{ }^{3}\right)$ & 0.327 & 0.67 \\
\hline Anti-Ro & -0.033 & 0.78 \\
\hline Anti-La & -0.125 & 0.29 \\
\hline \multicolumn{3}{|c|}{$\begin{array}{l}\text { BMI: body mass index, LDL: low density lipoprotein, } \\
\text { SBP: systolic blood pressure, DBP: diastolic blood } \\
\text { pressure, MAP: mean arterial pressure, PP: pulse } \\
\text { pressure, LVMI: left ventricular mass index, logCRP: } \\
\text { logarithmic C-reactive protein, WBC: white blood cell }\end{array}$} \\
\hline
\end{tabular}

pressure (SBP) and diastolic blood pressure (DBP) were $122 \pm 16 \mathrm{mmHg}$ and $76 \pm 9 \mathrm{mmHg}$, respectively. Demographical characteristics of the patients are listed in Table 1. Systemic involvement in our patients, including arthritis, was present in $12.5 \%$ of patients, skin lesions in $12.5 \%$, Raynaud's phenomenon in $13.9 \%$, muscle involvement in $1.4 \%$, lung involvement in $5.6 \%$, liver involvement in $5.6 \%$ and renal involvement in $1.4 \%$ of patients. Compared with the control subjects, patients with pSS had a higher mean PWV $(8.2 \pm 1.5 \mathrm{~m} / \mathrm{s}$ vs. $7.5 \pm 1.4 \mathrm{~m} / \mathrm{s} ; \mathrm{p}=0.01)$ despite similar age, BMI and blood pressure levels (Table 2). In pSS patients, correlation analysis showed that PWV was positively correlated with age, BMI, serum cholesterol, LDL and CRP lev-
Table 2. Comparison between Patients and Healthy Control.

\begin{tabular}{lccc}
\hline & $\begin{array}{c}\text { Healthy control } \\
(\mathrm{n}=68)\end{array}$ & $\begin{array}{c}\mathrm{pSS} \\
(\mathrm{n}=75)\end{array}$ & patients value \\
\hline Age $($ years $)$ & $51.9 \pm 9.9$ & $54.0 \pm 9.3$ & 0.195 \\
BMI $\left(\mathrm{kg} / \mathrm{m}^{2}\right)$ & $29.4 \pm 4.0$ & $28.9 \pm 4.8$ & 0.517 \\
SBP $(\mathrm{mmHg})$ & $124 \pm 18$ & $122 \pm 16$ & 0.440 \\
DBP $(\mathrm{mmHg})$ & $76 \pm 10$ & $76 \pm 9$ & 0.849 \\
MAP $(\mathrm{mmHg})$ & $94 \pm 12$ & $93 \pm 11$ & 0.629 \\
c-f PWV $(\mathrm{m} / \mathrm{s})$ & $7.5 \pm 1.4$ & $8.2 \pm 1.5$ & 0.010 \\
Total cholesterol (mg/dL) & $213 \pm 36$ & $203 \pm 32$ & 0.169 \\
LDL cholesterol (mg/dL) & $144 \pm 30$ & $121 \pm 32$ & 0.002 \\
CRP $(\mathrm{mg} / \mathrm{dL})$ & $0.33 \pm 0.29$ & $0.36 \pm 0.39$ & 0.748 \\
\hline SBP: systolic blood pressure, DBP: diastolic blood pressure, MAP: & &
\end{tabular}

Table 4. Linear Regression Analysis for Predictors of Arterial Stiffness Cf-PWV in Patients with Primary Sjögren's Syndrome.

\begin{tabular}{cccc}
\hline Variables & $\mathrm{t}$ & $\beta$ & $\mathrm{p}$ value \\
\hline $\begin{array}{c}\text { Mean Arterial Pressure } \\
\text { (mmHg) }\end{array}$ & 2,729 & .355 & .009 \\
Age (years) & 2,253 & .299 & .030 \\
LDL (mg/dL) & 2,129 & .260 & .039 \\
\hline
\end{tabular}

Included variables into the model as follows; age, body mass index, mean arterial pressure, pulse pressure, cholesterol, low-density lipoprotein (LDL), $\log \mathrm{C}$ - reactive protein.

els, SBP, DBP, mean arterial pressure (MAP), pulse pressure and left ventricular mass index (Table 3). Compared to pSS patients with a PWV $\leq 7.5$, pSS patients with a PWV $>7.5$ (the median value of healthy controls) were older, had higher SBP and DBP levels, and higher serum cholesterol, LDL and CRP levels. A multiple linear regression analysis revealed that arterial stiffness was associated with age (standardized beta $=0.299, \mathrm{p}=0.030$ ), MAP (standardized beta $=0.355, \mathrm{p}=0.009$ ) and LDL levels (standardized beta $=0.260$, $\mathrm{p}=0.039$ ) in pSS patients (Table 4).

\section{Discussion}

Previous studies demonstrated endothelial dysfunction and increased atherosclerosis in patients with Sjögren's syndrome, while there is no evidence in the literature of a relationship to arterial stiffness $(11,12)$. In the current study, we show that patients with Sjögren's syndrome have an increased arterial stiffness compared to the normal population. This finding indicates that, similar to patients with RA and SLE, patients with pSS may have an increased risk of cardiovascular disease.

Atherosclerosis is a multifactorial and systemic vascular disease involving the aging of the vessel wall that occurs over many years, and it is the most common cause of mortality and morbidity in the world. In recent times, atherosclerosis is increasingly considered an immune systemmediated process of the vascular system. One reason for the emergence of this idea is that there is an increased and ac- 
celerated rate of atherosclerosis in patients with autoimmune rheumatic diseases (AIRDs) such as RA and SLE, and these patients have higher rates of cardiovascular morbidity and mortality (13). This phenomenon can be attributed to traditional risk factors of atherosclerosis and the use of specific drugs, such as corticosteroids, but it might also be the result of other autoimmune and inflammatory mechanisms that are aggravated in AIRDs $(14,15)$.

Sjögren's syndrome is a connective tissue disease with a prevalent involvement of the exocrine glands. It can be seen alone or in combination with RA and SLE. Primary Sjögren's syndrome is usually characterized by circulatory RF and ANAs and polyclonal hypergammaglobulinemia. The disease is more prevalent in young females and it usually has a mild course. To our knowledge, there are no large, epidemiologic studies on survival and causes of death in Sjögren's syndrome. Theander et al. suggested that no increased all-cause mortality could be detected in patients with pSS compared with the general population. This study showed a standardized mortality ratio of 1.06 for cardiovascular (CV)-related deaths in a cohort of 265 Sjögren's syndrome patients with a median follow-up of seven years (16).

Similar to RA and SLE, Sjögren's syndrome has a known relationship with increased atherosclerosis, and cardiovascular disease risk has recently been investigated in patients with pSS. In the study conducted by Vaudo at al., 37 patients with pSS were compared with 35 healthy control subjects, and the authors found that patients with pSS had a higher intima media thickening than the controls at both the carotid (mean \pm SD $0.82 \pm 0.24 \mathrm{~mm}$ vs. $0.63 \pm 0.20 \mathrm{~mm} ; \mathrm{p}<$ $0.001)$ and femoral levels $(0.81 \pm 0.26 \mathrm{~mm}$ vs. $0.67 \pm 0.23$ $\mathrm{mm} ; \mathrm{p}<0.019)$, and also had a higher prevalence of carotid intima media thickening $(49 \%$ vs. $11 \%$ of controls; $\mathrm{p}<$ 0.001 ) (12). A multivariate analysis demonstrated that the presence of anti-SSA antibodies is an independent predictor of carotid artery thickening, while leukopenia was a predictor of both carotid and femoral artery thickening. Among the humoral factors examined, only anti-SSA antibodies were significantly associated with arterial thickening.

In another study conducted with 45 patients with pSS and 59 healthy control subjects, Gerli et al. showed that the flow-mediated vazodilatation (FMV) rates were similar in the two groups, while the nitroglycerin-mediated vazodilatation (NMV) rate was lower in the pSS group (11). In their study, the NMV rate was found to correlate with articular involvement, leucopenia, RF positivity and anti-Sjögren's syndrome B (SSB) positivity. In another study, Pirildar et al. found lower FMV rates in patients with pSS, while NMV rates were similar to controls (17). While the different findings of the two studies could originate from the characteristics of the patients, both studies suggest that chronic inflammation and immunological factors may be causes of endothelial dysfunction in pSS patients.

Our study is the first to study arterial stiffness in patients with pSS. We found that carotid-femoral PWV rates were significantly higher in patients with pSS than in the healthy population. The PWV rate was positively correlated with age, BMI, serum cholesterol, LDL and CRP levels, SBP, DBP, MAP, pulse pressure and left ventricular mass index. In multivariate analysis, predictors of the PWV rate included age, MAP and LDL level. Age, high BMI, hyperlipidemia and hypertension are factors which are known to correlate with accelerated arterial stiffness. It has been postulated that chronic inflammation plays a key role in inducing accelerated arterial stiffening. In the current study, there was a significant relationship between CRP levels and arterial stiffness. This finding supports the view that in patients with pSS, chronic inflammation may play a role in the pathogenesis of arterial stiffness. However, CRP was not a predictor, as determined by a multivariate analysis.

We could find no correlation between arterial stiffness and the leukocyte count or the presence of auto-antibodies. However, it is already known that these auto-antibodies have a definitive role in the pathogenesis of atherosclerosis. Pérez-De-Lis et al. reported that cardiovascular disease risk factors of patients with pSS include advanced age at diagnosis, hepatic involvement, central nervous system involvement, elevated CRP levels and low gamma globulin levels (18).

There are certain limitations associated with the current study. The number of patients with pSS taking medications such as steroids, antihypertensive drugs and statins, was rather high. Unfortunately, we could not stop these medications, and we could not exclude all the patients taking such drugs.

Risk factors associated with arterial stiffness in pSS patients are similar to the general population. Although there was a significant difference in arterial stiffness between patients with pSS and healthy controls, we cannot exclude the possibility that a higher PWV rate in pSS patients was caused, not by pSS itself, but by steroids, hypertension and dislipidemia.

\section{The authors state that they have no Conflict of Interest (COI).}

\section{References}

1. Koumaras C, Tzimou M, Stavrinou E, et al. Role of antihypertensive drugs in arterial 'de-stiffening' and central pulsatile hemodynamics. Am J Cardiovasc Drugs 12: 143-156, 2012.

2. Cernes R, Zimlichman R, Shargorodsky M. Arterial elasticity in cardiovascular disease: focus on hypertension, metabolic syndrome and diabetes. Adv Cardiol 45: 65-81, 2008.

3. Covic A, Gusbeth-Tatomir P, Goldsmith DJ. Arterial stiffness in renal patients: an update. Am J Kidney Dis 45: 965-977, 2005.

4. Blacher J, Protogerou AD, Henry O, et al. Aortic stiffness, inflammation, denutrition and type 2 diabetes in the elderly. Diabetes Metab 38: 68-75, 2012.

5. Kim YS, Sung YK, Choi CB, et al. The major determinants of arterial stiffness in Korean patients with rheumatoid arthritis are age and systolic blood pressure, not disease-related factors. Rheumatol Int 32: 3455-3461, 2012.

6. Pieringer H, Stuby U, Pohanka E, Biesenbach G. Augmentation index in patients with rheumatoid arthritis and ankylosing spondy- 
litis treated with infliximab. Clin Rheumatol 29: 723-727, 2010.

7. Colaci M, Giuggioli D, Manfredi A, et al. Aortic pulse wave velocity measurement in systemic sclerosis patients. Reumatismo 64: 360-367, 2012.

8. Soltész P, Dér H, Kerekes G, et al. A comparative study of arterial stiffness, flow-mediated vasodilation of the brachial artery, and the thickness of the carotid artery intima-media in patients with systemic autoimmune diseases. Clin Rheumatol 28: 655-662, 2009.

9. Peri Y, Agmon-Levin N, Theodor E, Shoenfeld Y. Sjögren's syndrome, the old and the new. Best Pract Res Clin Rheumatol 26: 105-117, 2012.

10. Gyöngyösi M, Pokorny G, Jambrik Z, et al. Cardiac manifestations in primary Sjögren's syndrome. Ann Rheum Dis 55: 450454, 1996.

11. Gerli R, Vaudo G, Bocci EB, et al. Functional impairment of the arterial wall in primary Sjögren's syndrome: combined action of immunologic and inflammatory factors. Arthritis Care Res (Hoboken) 62: 712-718, 2010.

12. Vaudo G, Bocci EB, Shoenfeld Y, et al. Precocious intima-media thickening in patients with primary Sjögren's syndrome. Arthritis Rheum 52: 3890-3897, 2005.

13. Kahlenberg JM, Kaplan MJ. Mechanisms of premature atherosclerosis in rheumatoid arthritis and lupus. Annu Rev Med 64: 249263, 2013.

14. Murdaca G, Colombo BM, Cagnati P, Gulli R, Spanò F, Puppo F. Endothelial dysfunction in rheumatic autoimmune diseases. Atherosclerosis 224: 309-317, 2012.

15. Peeters MA, Chizzolini C. Cardiovascular risk in autoimmune and inflammatory disorders. Rev Med Suisse 7: 867-871, 2011.

16. Theander E, Manthorpe R, Jacobsson LT. Mortality and causes of death in primary Sjögren's syndrome: a prospective cohort study. Arthritis Rheum 50: 1262-1269, 2004.

17. Pirildar T, Tikiz C, Ozkaya S, et al. Endothelial dysfunction in patients with primary Sjögren's syndrome. Rheumatol Int 25: 536539, 2005.

18. Pérez-De-Lis M, Akasbi M, Sisó A, et al. Cardiovascular risk factors in primary Sjögren's syndrome: a case-control study in 624 patients. Lupus 19: 941-948, 2010.

(C) 2016 The Japanese Society of Internal Medicine http://www.naika.or.jp/imonline/index.html 